\title{
Difference Equations on a Mesh Arising from a General Triangulation
}

\author{
By R. B. Kellogg
}

1. Introduction. Consider the boundary value problem

$$
\left\{\begin{aligned}
L u & \equiv-\left(p u_{x}\right)_{x}-\left(p u_{y}\right)_{y}+q u=f \\
u & =0 \text { on } \partial R
\end{aligned}\right.
$$

in a domain $R$ with polygonal boundary $\partial R$. The coefficients $p, q$ are assumed positive, bounded, and bounded away from 0 . It may be shown $[1$, p. 20$]$ that for $f$ square integrable, (1) has a unique "generalized solution" $u \in H_{0}{ }^{1}(R)$. (The notation is given in $\$ 2$.) It may be conjectured that if $p$ is smooth enough, $u$ has generalized derivatives of the second order and $\|u\|_{2} \leqq c\|f\|$. (In [3, p. 665] such a result is given if $\partial R$ is sufficiently smooth.)

We consider a class of finite difference approximations of (1),

$$
L_{1} v=f_{1},
$$

in which the mesh points of the approximation are the vertices of any triangulation of $R$ by acute triangles. These difference approximations were first considered by MacNeal [2] and include as a special case the usual 5 point difference approximation [5, chapter VI] to (1). It will be shown that, if $u \in H_{0}{ }^{1}(R) \cap H^{2}(R)$ is a solution of (1), a mean square norm of the error, $u-v$, is bounded by $c^{\prime} h\|u\|_{2}$, where $c^{\prime}$ is an explicit constant and $h$ is the maximum distance between neighboring mesh points.

This result contrasts with that of Nitsche and Nitsche [4] who obtain an $0\left(h^{2 / 5}\right)$ error estimate of the maximum norm of $u^{*}-v$ for more general second order elliptic equations and more special difference approximations. $\left(u^{*}\right.$ is a certain average of $u$.)

In the theories of heat conduction and neutron diffusion it is important to let $p, q$ be discontinuous. Let $p, q$ be smooth in the closure of each of a finite number of subdomains $R_{i}$ which make up the domain $R$. It is required that at each interface $\partial R_{i}$, the solution $u$ satisfies

$$
u, p \partial u / \partial n \text { continuous across } \partial R_{i},
$$

where $n$ is the normal vector at $\partial R_{i}$. If $u$ is twice differentiable in each $R_{i}$ and satisfies (1), (3), then for any $\phi \in H_{0}{ }^{1}(R)$,

$$
\iint_{R}\left\{p \phi_{x} u_{x}+p \phi_{y} u_{y}+q \phi u-f \phi\right\} d x d y=0
$$

so $u$ is the generalized solution whose existence is shown in [1]. The proofs in this paper are valid if $u \in H^{2}(T)$ where $T$ is any triangle in the triangulation which gives rise to the finite difference approximation (2). One may conjecture that the unique generalized solution $u \in H_{0}^{1}(R)$ of $(4)$ is in $H^{2}\left(R_{i}\right)$ for each subdomain $R_{i}$.

Received February 13, 1963, revised October 8, 1963. 
If this is true and if the $\partial R_{i}$ are polygons, then the results of this paper apply if the triangulation contains the polygons $\partial R_{i}$.

2. The Difference Equations. If $u$ is a function on a domain $U$, let $\|u, U\|=$ $\left\{\int_{U}|u|^{2} d x d y\right\}^{1 / 2}$. Define $\|u, U\|_{1}^{2}=\|u, U\|^{2}+\left\|u_{x}, U\right\|^{2}+\left\|u_{y}, U\right\|^{2}$, $\|u, U\|_{2}^{2}=\|u, U\|_{2}{ }^{2}+\left\|u_{x x}, U\right\|^{2}+\left\|u_{x y}, U\right\|^{2}+\left\|u_{y y}, U\right\|^{2} . H(U), H^{1}(U)$, $H^{2}(U)$ will denote the closure under the corresponding norms of the set of functions infinitely differentiable in a neighborhood of $\bar{U}$. These are Hilbert spaces. $H_{0}(U)$, $H_{0}{ }^{1}(U), H_{0}{ }^{2}(U)$ will denote the closed subspaces spanned by those infinitely differentiable functions which vanish outside a compact subset of $U$. The usual properties of these spaces will be assumed. In particular two simple inequalities should be noted. Namely

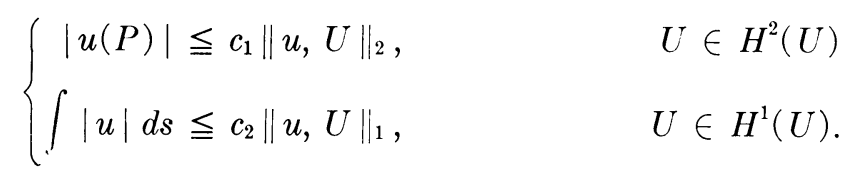

In these inequalities $U$ is a triangle and $c_{1}, c_{2}$ depend only on $U . P$ is a vertex of $U$ and, in the second inequality, the left side is a line integral taken along a line segment in $U$. From the first inequality it is seen that the $u(P)$ are meaningful quantities for our generalized solutions.

When $U=R$ we omit the $U$ in the above norms and spaces.

Let $J$ be a triangulation of $R$ such that the sides of the polygons $\partial R, \partial R_{i}$, all lie on the lines of $J$, and such that there are no obtuse triangles in $J$. Let $\mathcal{S}$ be the set of vertices of $J$, and let $S_{0}$ denote the points of $\delta$ lying inside $R$. Let there be $N$ points of $\delta_{0}$. We will say that two points of $\delta$ are neighbors if they are both vertices

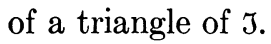

Let $\rho(P, Q)$ be the distance between points $P$ and $Q$, and let $h=\max \rho(P, Q)$, the maximum being taken over all neighbors $P, Q \in \mathcal{S}$. Let $c_{3}>1$ be a constant such that

$$
c_{3}^{-1} \leqq \rho(A, B) / \rho(C, D) \leqq c_{3}
$$

for each point $P \in \mathrm{s}$, where $A, B, C, D$ range over the set consisting of $P$ and its neighbors. The error bound will depend upon $c_{3}$, which may be thought of as a "local maximum mesh ratio". Let $h(P)$ be the maximum distance from $P$ to any one of its neighbors.

Let $\mathfrak{C}$ be the collection of all real valued mesh functions on $\mathcal{S}$, and let $\mathfrak{e}_{0} \subset \mathfrak{C}$ consist of those functions vanishing outside $S_{0}$. Then $\mathfrak{C}_{0}$ is an $N$ dimensional vector space and $L_{1}$ will be an $N$ by $N$ matrix acting on $C_{0}$. We introduce two inner products on $\mathfrak{C}_{0}$. If $\alpha, \beta \in \mathfrak{C}_{0}$, these are defined by

$$
\begin{aligned}
(\alpha, \beta) & =\sum h(P)^{2} \alpha(P) \beta(P), \\
(\alpha . \beta)_{1} & =(\alpha, \beta)+\sum_{1}(\alpha(P)-\alpha(Q))(\beta(P)-\beta(Q)) .
\end{aligned}
$$

The sum $\sum$ is taken over all $P \in \mathcal{S}$ and the sum $\sum_{1}$ is taken over all neighboring points $P, Q \in \delta$. The corresponding norms are denoted by $\|\alpha\|$ and $\|\alpha\|_{1}$.

Now let $\Im(P)$ be the set of triangles in $\Im$ with $P \in S_{0}$ as a vertex. Let $T \in J(P)$ 


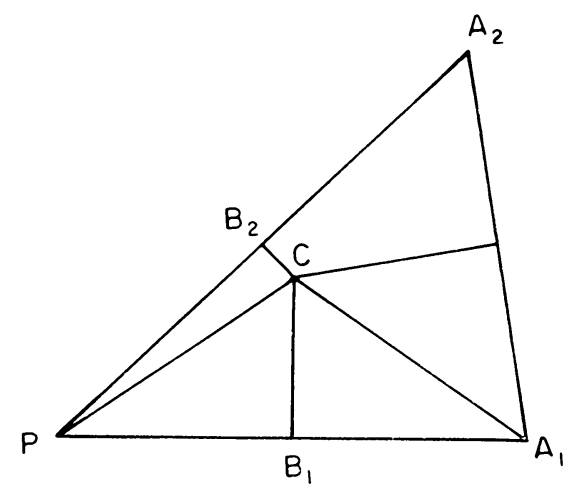

FIG. 1.

have vertices $P, A_{1}, A_{2}$, and let $B_{1} \mathrm{C}, B_{2} C$ be the perpendicular bisectors of $P A_{1}, P^{P} A_{2}$ (see figure 1 ). Since $T$ is acute, $C$ lies in $T$. Let $U$ denote the quadrilateral defined by $P B_{1} C B_{2}$. We define functions $a_{i}(P, T), b(P, T), f_{1}(P, T)$ by the equations

$$
\begin{array}{rlr}
a_{i}(P, T) & =\frac{1}{\rho\left(P, A_{i}\right)} \int_{B_{i} C} p d s, & i=1,2 \\
b(P, T) & =\iint_{U} q d x d y \\
f_{1}(P, T) & =\iint_{U} f d x d y .
\end{array}
$$

Then the difference approximation (2) arising from the triangulation $\Im$ is defined by

$$
\begin{aligned}
L_{1} v(P) & =\sum\left\{a_{1}(P, T)\left(v(P)-v\left(A_{1}\right)\right)+a_{2}(P, T)\left(v(P)-v\left(A_{2}\right)\right)+b(P, T) v(P)\right\} \\
& =\sum f_{1}(P, T),
\end{aligned}
$$

the sums being taken over $T \in J(P)$. Define functions $b(P), a(P, Q)$ by

$$
\begin{aligned}
b(P) & =\sum b(P, T), \quad T \in J(P) \\
a(P, Q) & =\left\{\begin{array}{lr}
a_{1}(P, T)+a_{2}\left(P, T^{\prime}\right), & Q \text { a neighbor of } P \\
0, & Q \text { not a neighbor of } P,
\end{array}\right.
\end{aligned}
$$

where $T, T^{\prime} \in J(P) \cap \Im(Q)$. Then (2) may be written

$$
L_{1} v(P) \equiv \sum a(P, Q)(v(P)-v(Q))+b(P) v(P)=f_{1}(P), \quad P \in \delta_{0} .
$$

By requiring $v \in \mathfrak{C}_{0}(7)$ is a system of $N$ equations in $N$ unknowns. $L_{1}$ is a symmetric, positive definite "Stieltjes" matrix. If $J(P)$ contains exactly 6 triangles for each $P, L_{1}$ is block 2 -cyclic, and the system (7) may be solved numerically by one of the variety of methods discussed in [5].

One could introduce an area weight at each $P \in$ s defined by $\sum_{T}|U|, T \in J(P)$, where $|U|$ is the area of the quadrilateral $U$, and use these weights to construct norms equivalent to $\|\alpha\|$, $\|\alpha\|_{1}$, but having more geometric meaning. The equivalence would be expressed with the constant $c_{3}$. 
3. Some Remainder Terms. In this section we give two approximation formulae with the error bounded in a form suitable for our later use. Let

$$
d=\max \{\rho(P, Q), P, Q \epsilon \bar{R}\} .
$$

Lemma 1. There is $a c_{4}>0$ depending only on $d$ such that, if $U$ is the quadrilateral $P B_{1} C B_{2}, u \in H^{2}(U)$, and $q$ is a nonnegative bounded function on $U$, then

$$
\left|\iint_{U} q u d x d y-u(P) \iint_{U} q d x d y\right| \leqq c_{4}(\sup q) h(P)^{2}\|u, U\|_{2} .
$$

Proof. It suffices to prove (8) for $u$ having continuous second derivatives. Referring to Figure 1 we may take $P$ to be the origin of coordinates and $A_{1}$ to lie on the positive $x$ axis. Using polar coordinates let the line $B_{1} C B_{2}$ be given by $r=$ $R(\theta), 0 \leqq \theta \leqq \alpha=$ the angle at $P$. For $(r, \theta) \in U$ one has

$$
u(r, \theta)-u(P)=\int_{\rho=0}^{r} u_{r}(\rho, \theta) d \rho=r u_{r}(r, \theta)-\int_{\rho=0}^{r} \rho u_{r r}(\rho, \theta) d \rho .
$$

Multiplying this by $r q$ and integrating over $U$ one finds that the left side of (8) is bounded by

$$
\begin{aligned}
\int_{\theta=0}^{\alpha} \int_{r=0}^{R(\theta)} & r q\left\{r u_{r}(r, \theta)-\int_{\rho=0}^{r} \rho u_{r r}(\rho, \theta) d \rho\right\} d r d \theta \\
\leqq & (\sup q) h(P) \iint_{U}\left|u_{r}\right| d x d y+(\sup q) h(P)^{2} \iint_{U}\left|u_{r r}\right| d x d y \\
& \leqq(\sup q) h(P)[1+h(P)]|U|^{1 / 2} \cdot\|u, U\|^{2}
\end{aligned}
$$

which proves (8) since $|U| \leqq h(P)^{2}$ and $1+h(P) \leqq 1+d$.

Lemma 2. There is a $c_{5}>0$ depending only on $c_{3}$ such that, if $V$ is the triangle $P C A_{1}, L$ is the line segment $B_{1} C, \eta$ is a unit vector pointing from $P$ to $A_{1}, u \in H^{2}(V)$, and $p$ is a nonnegative bounded function on $L$, then

$$
\left|\int_{L} p(\eta \cdot \nabla) u d s-\frac{u\left(A_{1}\right)-u(P)}{\rho\left(P, A_{1}\right)} \int_{L} p d s\right| \leqq c_{5}(\sup p) h(P)\|u, V\|_{2} .
$$

Proof. It suffices to prove (9) for $u$ having continuous second derivatives. Referring to Figure 1 we may take $B_{1}$ to be the origin of coordinates and $A_{1}$ to lie on the positive $x$ axis. Let $\rho\left(P, B_{1}\right)=\rho\left(A_{1}, B_{1}\right)=a, \rho\left(C, B_{1}\right)=b$. If $\xi(y)=$ $a(b-y) / b$ the line $C A_{1}$ contains the points $(\xi(y), y)$ and the line $C P$ contains the points $(-\xi(y), y), 0 \leqq y \leqq b$. The inequality (9) follows from the two inequalities

$$
\begin{aligned}
& \mid \int_{y=0}^{b} p(0, y)\left[u_{x}(0, y)-\left[\frac{u(\xi(y), y)-u(-\xi(y), y)}{2 \xi(y)}\right] d y \mid\right. \\
& \left|\int_{y=0}^{b} p(0, y)\left[\frac{u(\xi(y), y)-u(-\xi(y), y)}{2 \xi(y)}-\frac{u(a, 0)-u(-a, 0)}{2 a}\right] d y\right| \\
& \leqq c_{7}(\sup p) h(P)\|u, V\|_{2}
\end{aligned}
$$

where $c_{6}$ and $c_{7}$ are positive constants depending only on $c_{3}$. To prove (10) one 
may use Taylor's theorem with integral remainder term to bound the left side of (10) by

$$
\frac{1}{2}(\sup p) \int_{y=0}^{b} \int_{t=-\xi(y)}^{\xi(y)}\left|u_{x x}(t, y)\right| d t \leqq \frac{1}{2}(\sup p)|V|^{1 / 2}\|u, V\|_{2}
$$

and note that $|V| \leqq h(P)^{2}$.

The inequality (11) will now be proved. Define $\left|D^{2} u\right|=\left[u_{x x}^{2}+u_{x y}^{2}+u_{y y}^{2}\right]^{1 / 2}$. Then

$$
\begin{aligned}
\pm\left(u_{x}(\theta \xi(y), y)-u_{x}(\theta b, 0)\right)= \pm \int_{t=0}^{y} & \frac{d}{d t} u_{x}(\theta \xi(t), t) d t \\
& \leqq a^{-1}\left(a^{2}+b^{2}\right)^{1 / 2} \int_{t=0}^{y}\left|D^{2} u\right|(\theta \xi(t), t) d t .
\end{aligned}
$$

If this is integrated with respect to $\theta$ over $(-1,1)$ one obtains

$$
\begin{aligned}
\pm\left(\frac{1}{\xi(y)} \int_{-\xi(y)}^{\xi(y)} u_{x}(x, y) d x\right. & \left.-\frac{1}{b} \int_{-b}^{b} u_{x}(x, 0) d x\right) \\
& \leqq a^{-1}\left(a^{2}+b^{2}\right)^{1 / 2} \int_{t=0}^{y} \int_{s=-\xi(t)}^{\xi(t)} \frac{1}{\xi(t)}\left|D^{2} u\right|(s, t) d s d t .
\end{aligned}
$$

After multiplying both sides of $(12)$ by $p(0, y)$, integrating with respect to $y$ over $(0, b)$, and interchanging the order of the $y$ and $t$ integrations, one finds that the left side of (11) is bounded by

$$
(\sup p) b a^{-2}\left(a^{2}+b^{2}\right)^{1 / 2} \iint_{V}\left|D^{2} u\right| d x d y \leqq(\sup p) c_{5}|V|\|u, V\|_{2} .
$$

This proves (11) because $|y| \leqq h(P)^{2}$.

4. The Discretization Error. For our error bounds we assume there exists a $c_{6}>1$ such that in the closure of $R$,

$$
1 / c_{6} \leqq p(x, y), \quad q(x, y) \leqq c_{6} .
$$

We also define a constant $c_{7}$ by the condition that no $P \in \mathcal{S}$ has more than $c_{7}$ neighbors.

Lemma 3. There is a $c_{8}$ depending on $c_{3}, c_{6}$, and $c_{7}$ such that

$$
c_{8}^{-1}\|\alpha\|_{1} \leqq\left\{\sum \alpha(P) L_{1} \alpha(P)\right\}^{1 / 2} \leqq c_{8}\|\alpha\|_{1}
$$

for any $\alpha \in \mathcal{C}_{0}$, the sum being taken over $P \in \mathcal{S}$.

Proof. One has

$$
\sum \alpha(P) L_{1} \alpha(P)=\frac{1}{2} \sum_{1} a(P, Q)(\alpha(P)-\alpha(Q))^{2}+\sum b(P) \alpha(P)^{2} .
$$

The proof follows easily from (15).

$L_{1}$ is symmetric and (15) shows that it is positive definite. Hence we define an inner product on $\mathrm{C}_{0}$ by $(\alpha, \beta)^{\prime}=\sum \alpha(P) L_{1} \beta(P)$, and denote the corresponding norm by $\|\alpha\|^{\prime}$.

Theorem 1. Let $u \in H_{0}{ }^{1} \cap H^{2}$ be a solution of (1), and let $v \in \mathfrak{C}_{0}$ be the corresponding solution of (2). Then there is a constant $c_{9}$ depending only on $c_{3}, c_{6}, c_{7}$, and $d$, 
such that, if $e \in \mathfrak{C}_{0}$ is defined by $e(P)=u(P)-v(P)$, then

$$
\|e\|_{1} \leqq h c_{9}\|u\|_{2} \text {. }
$$

Proof. Using (14), one has

$$
\|e\|_{1} \leqq c_{8}^{2}\|e\|^{\prime}
$$

Hence the theorem follows from the inequality

$$
\left|(e, e)^{\prime}\right| \leqq h c_{10}\|e\|_{1}\|u\|_{2},
$$

where $c_{10}$ depends only on $c_{3}, c_{6}, c_{7}$, and $d$. One has $L_{1} e=L_{1} u-f_{1}$. Because $u \in H^{2}$, one has, referring to Figure 1 ,

$$
f_{1}(P)=\sum\left\{\int p \frac{d u}{d n} d s+\iint q u d x d y\right\},
$$

the sum being taken over all triangles $T \in J(P)$; the line integral is taken over the line segments $B_{1} C B_{2}$, and the area integral is taken over the quadrilateral $P B_{1} C B_{2}$. Analogous to (15), a calculation gives

$$
(e, e)^{\prime}=\frac{1}{2} \sum_{1}[e(P)-e(Q)] E(P, Q)+\sum e(P) F(P)
$$

where

$$
E\left(P, A_{1}\right)=\frac{u(P)-u\left(A_{1}\right)}{\rho\left(P, A_{1}\right)} \int p d s-\int p \frac{d u}{d n} d s
$$

the line integral being taken over $B_{1} C$ and the corresponding perpendicular bisector on the other side of $P A_{1}$ (see Figure 1), and

$$
F(P)=u(P) \iint q d x d y-\iint u p d x d y,
$$

the area integrals being taken over all the quadrilaterals $P B_{1} C B_{2}$ of triangles $T \in J(P)$. Using Lemmas 1 and 2 we obtain

$$
\begin{aligned}
\left|(e, e)^{\prime}\right| & \leqq \frac{1}{2} c_{5} c_{6} \sum_{1} h(P)\|u, T\|_{2}|e(P)-e(Q)|+c_{4} c_{6} \sum h(P)^{2}\|u, T\|_{2} \mid e(P) \\
& \leqq c_{11} h\left\{\sum|e(P)-e(Q)|^{2}\right\}^{1 / 2}\|u\|_{2}+c_{11} h\left\{\sum h(P)^{2} e(P)^{2}\right\}^{1 / 2}\|u\|_{2} \\
& \leqq 2 c_{11} h\|e\|_{1}\|u\|_{2},
\end{aligned}
$$

which proves the theorem.

It is easily seen that the proof remains valid if $u \in H^{2}(T)$ for each triangle $T$ of $J$.

To extend this result to the case $q \geqq 0$ it seems necessary to make further restrictions on the triangulation. The first requirement is

(A) There is a $c_{12}>1$ such that whenever $A, B, C, D \in \mathcal{S}$ and $A$ and $B$ are neighbors and $C$ and $D$ are neighbors, one has

$$
\left(c_{12}\right)^{-1} \leqq \rho(A, B) / \rho(C, D) \leqq c_{12} .
$$

To state the second condition, let a line $\lambda$ of $J$ be a sequence $\left\{P_{1}, P_{2}, \cdots, P_{n}\right\}$ of points of $S$ such that $P_{i}$ is a neighbor of $P_{i+1}, 1 \leqq i<n$, define the ends of $\lambda$ to be the points $P_{1}, P_{n}$, and define the length of $\lambda$ to be $\sum \rho\left(P_{i}, P_{i+1}\right), 1 \leqq i<n$. 
The second condition is

(B) $\delta$ may be written as a union of a set of lines $\lambda$ such that no two lines have a point in common and each line has a least one endpoint on $\partial R$. Given such a decomposition of $\mathcal{S}$, let $c_{13}$ denote the maximum length of the lines $\lambda$ in the decomposition.

We also assume that there exists a $c_{14}>1$ such that in the closure of $R$,

$$
\left\{\begin{aligned}
p(x, y), q(x, y) & \leqq c_{14} \\
q(x, y) & \geqq 0 \\
p(x, y) & \geqq 1 / c_{14}
\end{aligned}\right.
$$

Then Lemma 3 is easily extended as follows.

Lemma 4. Suppose J satisfies (A) and (B) and suppose (18) holds. Then there is a $c_{15}$ depending on $c_{7}, c_{12}, c_{13}$, and $c_{14}$, such that

$$
\left(c_{15}\right)^{-1}\|\alpha\|_{1} \leqq\left\{\sum \alpha(P) L_{1} \alpha(P)\right\}^{1 / 2} \leqq c_{15}\|\alpha\|_{1}
$$

for any $\alpha \in \mathcal{C}_{0}$, the sum being taken over $P \in \mathcal{S}$.

Proof. Let $\lambda=\left\{P_{1}, \cdots, P_{n}\right\}$ be one of the lines of (B). Then

$$
\left|\alpha\left(P_{j}\right)\right| \leqq \sum\left|\alpha\left(P_{i+1}\right)-\alpha\left(P_{i}\right)\right| \leqq\left[(n-1) \sum\left(\alpha\left(P_{i+1}\right)-\alpha\left(P_{i}\right)\right)^{2}\right]^{1 / 2},
$$

$1 \leqq i<n$.

Hence

$$
\sum(n-1)^{-2} \alpha\left(P_{i}\right)^{2} \leqq \sum\left(\alpha\left(P_{i+1}\right)-\alpha\left(P_{i}\right)\right)^{2}, \quad 1 \leqq i<n .
$$

Now for any $j, 1 \leqq j \leqq n$,

$$
c_{13} \geqq \sum \rho\left(P_{i}, P_{i+1}\right) \geqq(n-1) h\left(P_{j}\right)\left(c_{12}\right)^{-1} \text {. }
$$

Hence

$$
\sum h\left(P_{i}\right)^{2} \alpha\left(P_{i}\right)^{2} \leqq\left(c_{12} c_{13}\right)^{2} \sum\left(\alpha\left(P_{i+1}-\alpha\left(P_{i}\right)\right)^{2}, \quad 1 \leqq i<n .\right.
$$

The left sum may be extended over $1 \leqq i \leqq n$. This is obvious if $\alpha\left(P_{n}\right)=0$, and if $\alpha\left(P_{1}\right)=0$ the same argument may be applied to the lines $\lambda$ ordered in the other direction. Summing this over all lines $\lambda$ of the decomposition and using (15),

$$
(\alpha, \alpha) \leqq 4 c_{12}^{3} c_{13}^{2} \sum \alpha(P) L_{1} \alpha(P) .
$$

The rest of the proof follows that of Lemma 3.

Using this lemma the following theorem may be proved in the same manner as Theorem 1.

Theorem 2. Assume (A), (B), and (18). Then there is a constant $c_{16}$ depending only on $c_{7}, c_{12}, c_{13}, c_{14}$, and $d$, such that if $u \in H_{0}{ }^{1} \cap H^{2}$ is a solution of (1) and $v \in \mathfrak{C}_{0}$ is the corresponding solution of $(2)$, and $e(P)=u(P)-v(P)$, then

$$
\|e\|_{1} \leqq h c_{16}\|u\|_{2} \text {. }
$$

Bettis Atomic Power Laboratory

Pittsburgh, Pennsylvania 
1. J. L. Lions, Equations Differentielles Operationelles, Springer, Berlin, 1961.

2. R. H. MacNeal, "An asymetrical finite difference network," Quart. Appl. Math., v. 12, 1953, p. 295-310.

3. L. Nirenberg, "Remarks on strongly elliptic partial differential equations," Comm. Pure Appl. Math., v. 8, 1955, p. 649-674.

4. J. Nitsche \& J. C. C. Nitsche, "Error estimates for the numerical solution of elliptic differential equations," Arch. Rational Mech. Anal., v. 5, 1960, p. 293-306.

5. R. S. VARGA, Matrix Iterative Analysis, Prentice-Hall, New York, 1962. 